\title{
Características sensoriales de papas fritas en bastones
}

\author{
J. Trinchero ${ }^{1}$, M. Monti ${ }^{1}$, P. Ceroli ${ }^{2}$
}

\begin{abstract}
RESUMEN
Se caracterizaron sensorialmente papas fritas en bastones de las variedades Frital INTA, Kennebec, Spunta y Shepody aplicando el análisis descriptivo cuantitativo. Los descriptores desarrollados fueron longitud y firmeza del bastón, color externo, dureza, crocantez, harinosidad, humedad, granulosidad y aceitosidad residual. Los atributos olor y sabor no se evaluaron por no considerarse característico de la papa sino del aceite de fritura. Los mismos se cuantificaron con escalas no estructuradas ancladas en los extremos. Los datos se analizaron mediante análisis de varianza (ANOVA) de un factor, test de Tukey y análisis de componentes principales (ACP). La variedad de mayor intensidad de color externo fue Spunta. Shepody y Spunta fueron las de mayor longitud de bastón. En firmeza y crocantez se encontró diferencia significativa entre Spunta y Frital INTA, siendo ésta última la más firme y crocante. Frital INTA obtuvo mayor puntaje de dureza y granulosidad aunque no se observaron diferencias significativas entre las variedades. Frital INTA presentó los valores más elevados de harinosidad. Spunta mostró los mayores valores de humedad y aceitosidad residual y Frital INTA los menores. En el ACP los primeros dos componentes explicaron el $97,98 \%$ de la variabilidad total. Frital INTA se asoció con dureza, granulosidad, crocantez, firmeza y harinosidad. Spunta se asoció con humedad, aceitosidad residual y color. Kennebec mostró valores promedio, se asoció con humedad, dureza, granulosidad y aceitosidad residual. Shepody se asoció con la longitud de bastones y con color externo. Se concluye que la metodología aplicada permitió evaluar sensorialmente a las distintas variedades. Frital INTA se caracteriza por atributos relacionados con la textura. Por otro lado, Spunta por atributos como mayor longitud de bastón, humedad, aceitosidad residual y color más intenso. Kennebec y Shepody muestran un comportamiento intermedio.
\end{abstract}

Palabras clave: papas fritas, evaluación sensorial, perfiles sensoriales.

\section{Sensory characteristics of french fries potatoes}

\begin{abstract}
French fries of the varieties Frital INTA, Kennebec, Spunta and Shepody were sensory characterized with quantitative descriptive analysis. The descriptors used were length and firmness of sticks, external color, hardness, crunchiness, mealiness, moistness, graininess and oil residual. The descriptors were quantified with scales not structured anchored in the ends. Analysis of variance of one factor, Tukey's test and principal component analysis (PCA) were applied. Spunta was of more intensity external color. Shepody and Spunta had longer sticks. Firmness and crunchiness of Spunta and Frital INTA were both significantly different. The most firm and crunchy of all varieties was Frital INTA, wich also had the highest score for

\footnotetext{
${ }^{1}$ Facultad de Ciencias Agrarias (UN Mar del Plata) Ruta Nacional 226 Km 73,5, (B7620ZAA) Balcarce, Argentina. Correo electrónico: itrinchero@balcarce.inta.gov.ar, cmonti@balcarce.inta.gov.ar

${ }^{2}$ EEA INTA Balcarce. Ruta Nacional 226 Km 73,5, (B7620ZAA) Balcarce, Argentina.

Correo electrónico: paolaceroli@yahoo.com.ar
} 
hardiness, graininess and mealiness, though no significant differences were observed among varieties. The highest values of moisture and oil residual were shown by Spunta while Frital INTA showed the lowest. The first two components explained $97.98 \%$ of the total variability in PCA. Frital INTA was associated with hardness, graininess, crunchiness, firmness and mealiness but Spunta with moistness, oil residual and color. Kennebec had average values and became associated with moistness, hardness, graininess and oil residual. Shepody was associated with length sticks and external color. The quantitative descriptive analysis of the sensory analysis applied in this study allowed the characterization of different potato varieties. Frital INTA is characterized by attributes related with texture. On the other hand, Spunta is characterized by attributes such as longer sticks, moistness, oil residual and more intense colour. Kennebec and Shepody show an intermediate behavior.

Key words: french fries, sensorial evaluation, sensorial profile.

\section{Introducción}

La papa, Solanum tuberosum L., constituye uno de los principales alimentos en la canasta familiar. Se consume principalmente como producto natural sin procesos industriales. Sin embargo, en nuestro país su empleo como producto procesado está adquiriendo cada vez más importancia. Actualmente se destaca la producción de papas prefritas congeladas, papas fritas en hojuelas $y$ productos prefritos formulados con puré deshidratado.

El crecimiento de la producción industrial entre 1.996 y 2.003 fue consecuencia de importantes inversiones extranjeras registradas en el sector, las que a su vez requirieron un proceso de reconversión en la producción primaria para satisfacer las exigencias de calidad de la industria. La demanda de papas con características específicas requeridas por las industrias procesadoras hace necesario la caracterización de las variedades para los distintos usos. Las características de calidad de los tubérculos dependen del componente genético de las variedades, de las condiciones ambientales y el manejo agronómico.

Dentro de los atributos que caracterizan la calidad culinaria de la papa se consideran la textura, el olor, el sabor y el color. La textura es uno de los parámetros más importantes para determinar la calidad sensorial del tubérculo (Ross y Scanlon, 2004).
La descripción de las propiedades sensoriales de un producto se puede realizar a través de los perfiles sensoriales, donde se considera el orden de percepción de los atributos y se asigna un valor de intensidad para cada uno (IRAM 20019, 2004). Es importante tener en cuenta que los individuos son el instrumento de medida, por lo tanto es necesario seleccionarlos, entrenarlos y confirmar su eficacia, evaluando la consistencia de sus respuestas y la habilidad en distinguir diferencias entre muestras (Montauto-Graña et al., 2002).

El objetivo de este trabajo fue obtener la caracterización sensorial de papas fritas en bastones de diferentes variedades aplicando la metodología del análisis descriptivo cuantitativo.

Los resultados permitirán contribuir a ampliar el conocimiento de las características sensoriales de los productos elaborados a partir de papa, predecir la calidad sensorial del producto a través del análisis de la materia prima y posibilitar el uso alternativo de variedades de papa para obtener productos con características sensoriales similares.

\section{Materiales y métodos}

\section{Muestras}

Se utilizaron cuatro variedades de papa: Frital INTA, Kennebec, Spunta y Shepody, cosechadas en el Sudeste Bonaerense en febrero del 2.004. Fueron almacenadas a $10{ }^{\circ} \mathrm{C}$ hasta cinco días previos al análisis y se acondicionaron a temperatura ambiente hasta el momento del 
mismo. Las muestras fueron de $5 \mathrm{~kg}$ de papa por cada variedad.

\section{Evaluadores}

Se seleccionó y entrenó en la metodología de perfiles descriptivos de papa un panel de nueve miembros pertenecientes a la Estación Experimental Agropecuaria (EEA), INTA, Balcarce (Trinchero et al., 2006).

\section{Lugar del ensayo}

El ensayo se realizó en el área de evaluación sensorial del Laboratorio de Calidad de Papa y Tecnología de Poscosecha, EEA, INTA, Balcarce.

\section{Preparación de las muestras para freír}

A partir de la muestra de $5 \mathrm{~kg}$ se tomó una submuestra de 10 tubérculos que fueron lavados, pelados y cortados en bastones $(10 \times 10 \mathrm{~mm})$. Se eligieron los cuatro bastones centrales de cada tubérculo. La fritura se realizó en 15 litros de aceite de girasol con porciones de 250 gramos de bastones, durante 5 minutos a $180^{\circ} \mathrm{C}$.

Las muestras fueron servidas en platos codificados con números de tres dígitos al azar.

\section{Evaluación sensorial}

Para la evaluación sensorial de las muestras se utilizó la metodología de análisis descriptivo cuantitativo (Stone y Sidel, 1993; van Oirschot et al., 2003), utilizando escalas no estructuradas, de $10 \mathrm{~cm}$ de longitud, ancladas en los extremos con términos descriptivos. La generación de descriptores, la forma de evaluarlos y las escalas a utilizar se desarrollaron en las sesiones de trabajo grupal durante el entrenamiento del panel. Se analizó la eficacia de los panelistas mediante tres repeticiones, evaluando la reproducibilidad de los mismos $(p \geq 0,05)$ y su capacidad discriminatoria $(\mathrm{p} \leq 0,50)$ (MontautoGraña et al., 2002) aplicando un análisis de varianza (ANOVA) de dos factores (muestra y sesión) para cada evaluador y cada atributo (Statistica 5.1).

\section{Análisis de las muestras}

Debido a que en ensayos previos se comprobó que los panelistas podían evaluar en cada sesión hasta cuatro variedades se utilizó un diseño de bloques completos aleatorizados. Este diseño incluye los panelistas como factor de bloqueo y se realizaron cuatro repeticiones por variedad.

Los datos obtenidos se analizaron mediante ANOVA de un factor y con pruebas de comparaciones múltiples de Tukey $(p<0,05)$.

Por tratarse de datos multidimensionales y dadas las interrelaciones entre las variables, se realizó un análisis de componentes principales (ACP) utilizando una matriz de covarianza con el valor medio de los nueve panelistas por cada atributo (Peña, 2002). El análisis estadístico se llevó a cabo con el programa $\mathrm{R}$ 1.9.0.

\section{Resultados y discusión}

\section{Entrenamiento de los evaluadores}

Los descriptores generados por los evaluadores fueron: longitud del bastón, firmeza, color externo, dureza, crocantez, harinosidad, granulosidad, humedad y aceitosidad residual. Se definió la forma de evaluación de cada uno y se construyeron las escalas correspondientes (Figura 1). La firmeza del bastón se determinó flexionando con los dedos y el resto de los parámetros de textura se evaluaron tomando como referencia la norma IRAM 20013, 1998. Los atributos olor y sabor no se evaluaron por considerarse afectados por el aceite de fritura.

El total de evaluadores discriminaron aceitosidad residual y longitud de bastones en todas las sesiones. Seis de ellos obtuvieron un valor significativo en firmeza y humedad; cinco en harinosidad, color externo y granulosidad, cuatro en dureza y tres en crocantez de la corteza. Mostraron tener buena reproducibilidad en las distintas sesiones. Nueve reprodujeron sus valores en aceitosidad residual, longitud del basón, firmeza, color, dureza y harinosidad; ocho en crocantez y humedad y siete en granulosidad. 
EVALUACIÓN SENSORIAL DE PAPAS FRITAS EN BASTONES

NOMBRE Y APELLIDO:

FECHA:

Longitud de bastones

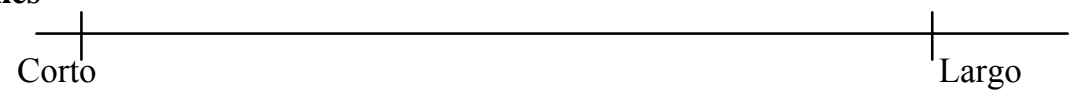

Firmeza del bastón:

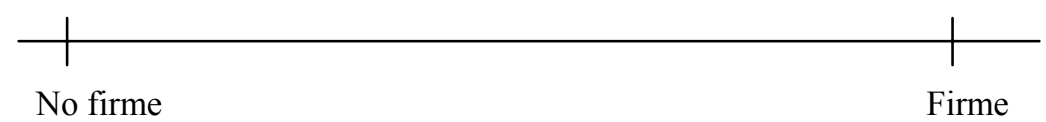

Color externo

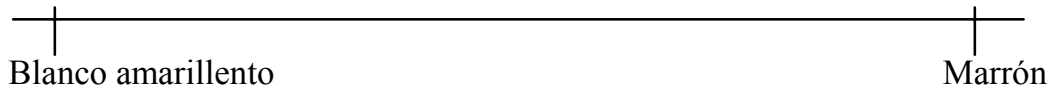

Dureza

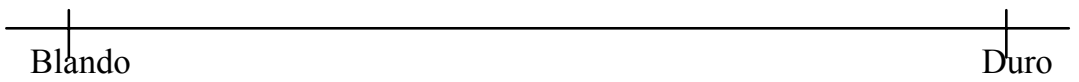

Crocantez

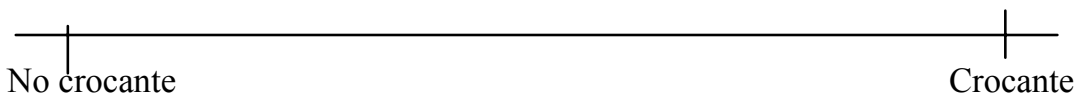

Harinosidad

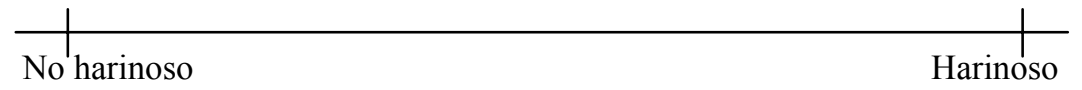

\section{Granulosidad}

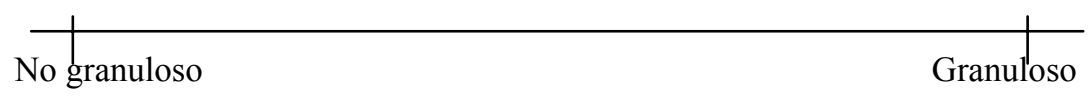

Humedad

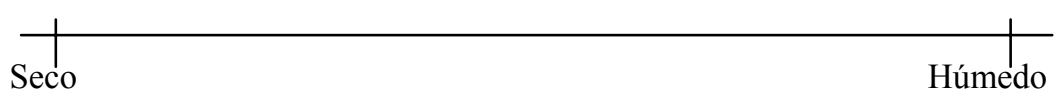

Aceitosidad Residual

No aceitoso

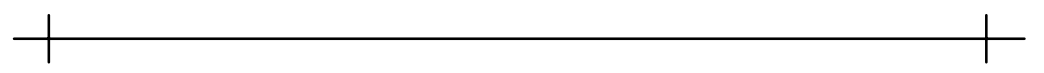

Muy aceitoso

\section{Observaciones:}

Figura 1. Planilla de evaluación sensorial de papas fritas en bastones 


\section{Evaluación de las muestras}

La Tabla 1 muestra los valores promedios de los evaluadores para cada descriptor $y$ las diferencias significativas entre las variedades.
La Figura 2 representa los perfiles sensoriales de las variedades obtenidos a partir de dichos promedios.

Tabla 1. Valores promedio de los descriptores por variedad.

\begin{tabular}{lcccc}
\hline ATRIBUTOS & KENNEBEC & SPUNTA & SHEPODY & FRITAL \\
\hline LONG.BAST. & $3,65 \mathrm{a}$ & $7,83 \mathrm{~b}$ & $7,51 \mathrm{bc}$ & $3,05 \mathrm{a}$ \\
FIRMEZA BAST. & $4,85 \mathrm{ab}$ & $3,08 \mathrm{a}$ & $4,44 \mathrm{ab}$ & $5,71 \mathrm{~b}$ \\
COLOR EXT & $3,14 \mathrm{a}$ & $4,88 \mathrm{~b}$ & $1,69 \mathrm{c}$ & $2,96 \mathrm{ad}$ \\
DUREZA & $5,09 \mathrm{a}$ & $4,08 \mathrm{a}$ & $4,38 \mathrm{a}$ & $5,43 \mathrm{a}$ \\
CROCANTEZ & $3,18 \mathrm{ab}$ & $2,11 \mathrm{a}$ & $2,85 \mathrm{ab}$ & $3,68 \mathrm{~b}$ \\
HARINOSIDAD & $4,62 \mathrm{ab}$ & $3,52 \mathrm{a}$ & $5,11 \mathrm{~b}$ & $5,44 \mathrm{~b}$ \\
HUMEDAD & $5,42 \mathrm{a}$ & $7,26 \mathrm{~b}$ & $4,28 \mathrm{ac}$ & $3,33 \mathrm{c}$ \\
GRANULOSIDAD & $5,44 \mathrm{a}$ & $4,76 \mathrm{a}$ & $4,54 \mathrm{a}$ & $6,09 \mathrm{a}$ \\
ACEITOSIDAD RESIDUAL & $5,51 \mathrm{a}$ & $7,15 \mathrm{~b}$ & $4,34 \mathrm{ac}$ & $3,65 \mathrm{c}$ \\
\hline
\end{tabular}

Valores con distintas letras indican diferencias significativas de cada atributo entre las distintas variedades según el test de Tukey $(\mathrm{p}<0.05)$.

\section{Longitud}

La longitud es una característica de las variedades y puede estar afectada por las condiciones del cultivo. Shepody y Spunta presentaron la mayor longitud de bastón. Aplicando el test de comparaciones múltiples de Tukey se encontró diferencia significativa ( $\mathrm{p}<$ 0,05) entre Kennebec y Spunta; Kennebec y Shepody; Frital INTA y Spunta; Frital INTA y Shepody.

\section{Firmeza}

La firmeza del bastón es una característica de textura que se relaciona con el contenido de almidón y la estructura celular (Shomer y Levy, 1988). Se observó diferencia significativa ( $\mathrm{p}<$ 0,01 ) entre Spunta y Frital INTA, siendo ésta última la más firme. Kennebec, Shepody y Spunta, no mostraron diferencias significativas.

\section{Color externo}

La variedad que presentó mayor intensidad de color externo fue Spunta. Se encontró diferencia significativa $(p<0,01)$ entre Kennebec y Spunta; Kennebec y Shepody; Spunta y Shepody; Spunta y Frital INTA; Frital INTA y Shepody.

\section{Dureza y granulosidad}

En los atributos de dureza y granulosidad no se observaron diferencias significativas entre las variedades.

\section{Crocantez}

Se encontró diferencia significativa $(p=0,04)$ entre Spunta y Frital INTA, siendo esta última la más crocante. La crocantez depende del contenido de materia seca del tubérculo crudo y del tiempo y temperatura de fritado (Kelly y Scanlen, 2004).

\section{Harinosidad}

Frital INTA y Shepody presentaron los valores más elevados de harinosidad $\mathrm{y}$ difieren significativamente de Spunta $(p=0,02)$.

\section{Humedad y aceitosidad residual}

Spunta presentó los mayores valores de humedad y aceitosidad residual y Frital INTA los menores. Se encontró diferencia significativa $(\mathrm{p}<$ 0,01) entre Kennebec y Spunta; Kennebec y Frital INTA; Spunta y Shepody; Spunta y Frital INTA. 
La humedad después de la cocción de las papas fritas se correlaciona negativamente con el contenido de almidón (Quirien et al., 2003). El contenido de aceite en la papa frita se relaciona con el contenido de almidón y la materia seca del material crudo (Kita, 2002). Las variedades con alto contenido de materia seca tienen baja absorción de aceite (Kabira, 2000).

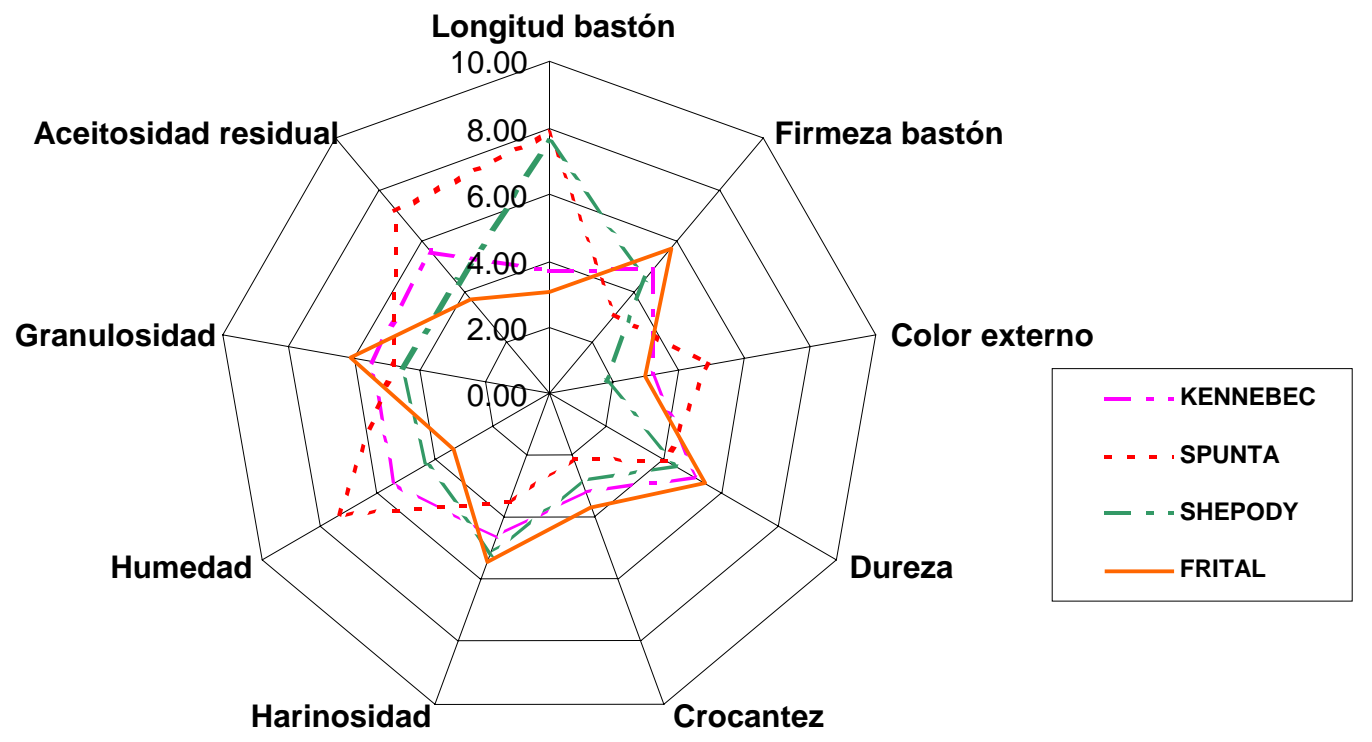

Figura 2. Perfiles sensoriales de las variedades obtenidos a partir de los valores promedio del conjunto de panelistas.

\section{Análisis de componentes principales}

Se aplicó el ACP tomando los valores medios de las variables de cada variedad del total de evaluadores (Tabla 2). Los dos primeros componentes explican el 97,98\% de la variabilidad total. El CP1 explica el $74,78 \%$ y el CP2 el 14\% de la varianza total de las variables. La contribución de los nueve atributos a los dos primeros componentes se muestra en la Tabla 2 mediante sus coeficientes de correlación.

El CP1 está definido positivamente con humedad, aceitosidad residual y longitud del bastón y negativamente con firmeza del bastón, dureza, crocantez, harinosidad, granulosidad y humedad. El CP2 está definido principalmente por color externo.
Tabla 2.Correlación entre atributos y los dos primeros componentes:

\begin{tabular}{lcc}
\hline ATRIBUTOS & CP1 & CP2 \\
\hline Long. bastón & 0,86 & 0,50 \\
Firmeza & $-1,00$ & 0,02 \\
Color externo & 0,59 & $-0,74$ \\
Dureza & $-0,94$ & $-0,04$ \\
Crocantez & $-1,00$ & $-0,04$ \\
Harinosidad & $-0,90$ & 0,43 \\
Humedad & 0,90 & $-0,42$ \\
Granulosidad & $-0,80$ & $-0,53$ \\
Aceitosidad residual & 0,88 & $-0,46$ \\
\hline
\end{tabular}


Mediante el porcentaje de reconstrucción de cada una de las variables utilizando los dos primeros componentes (Tabla 3), se observó que todas las variables se encuentran bien reconstruidas indicando buena representación en el plano de los dos primeros componentes.

Proyectando las variedades a los dos ejes y asociándolas con los atributos sensoriales (Figura 3) se observó lo siguiente: Las papas fritas de Frital INTA están asociadas con dureza, granulosidad, crocantez, firmeza y harinosidad; sus bastones son cortos, no húmedos y de poca aceitosidad residual.

Las papas fritas de Spunta se asocian con humedad, aceitosidad residual y color externo amarillo; sus bastones son largos y poco firmes. Las papas fritas de Kennebec se encuentran
Tabla 3. Porcentaje de Reconstrucción de cada variable utilizando los dos primeros componentes.

\begin{tabular}{lc}
\hline Longitud del bastón & $99,57 \%$ \\
Firmeza & $99,99 \%$ \\
Color externo & $89,12 \%$ \\
Dureza & $99,99 \%$ \\
Crocantez & $99,99 \%$ \\
Harinosidad & $99,99 \%$ \\
Humedad & $98,27 \%$ \\
Granulosidad & $92,76 \%$ \\
Aceitosidad residual & $98,36 \%$ \\
\hline
\end{tabular}

cerca del promedio. Se asocian con humedad, dureza, granulosidad y aceitosidad residual. La variedad Shepody se asocia con la longitud del bastón y por color externo blanco.

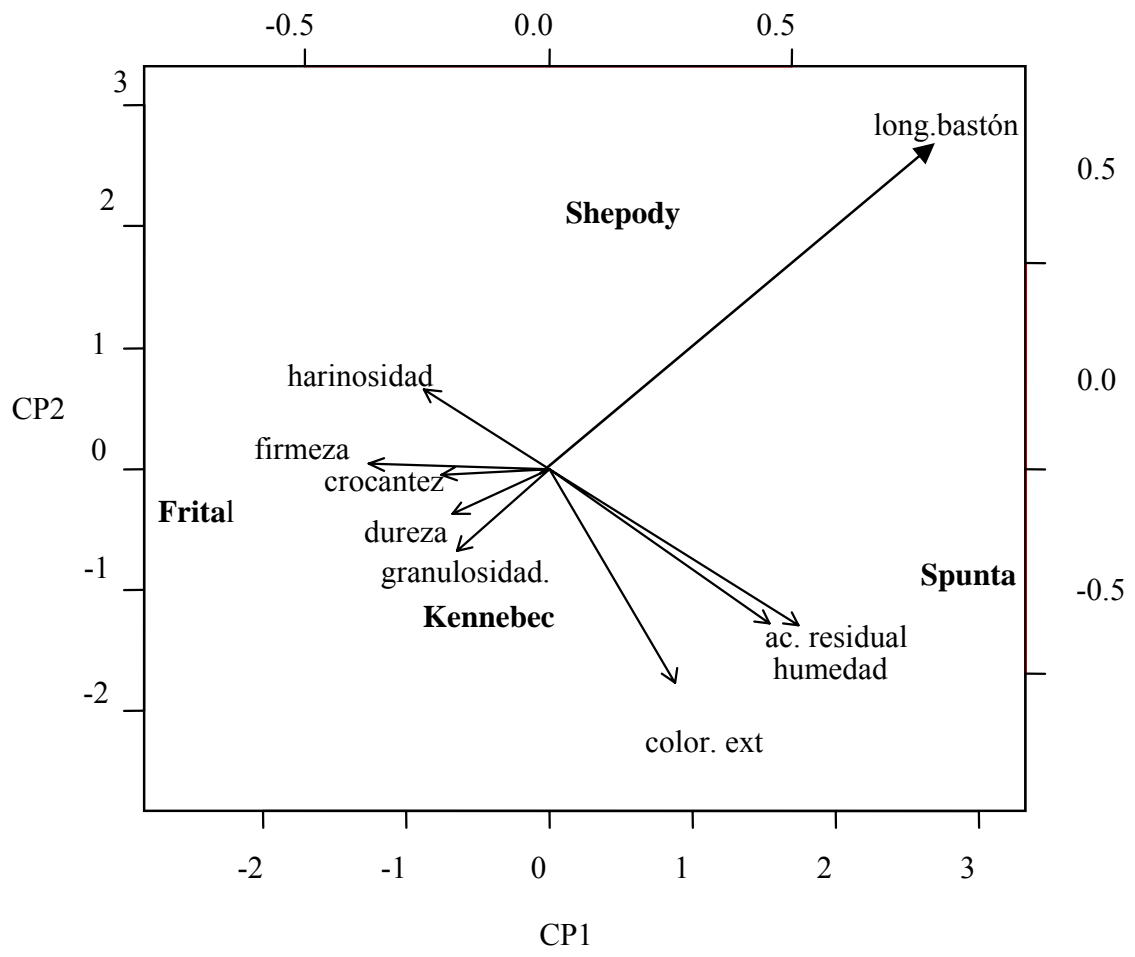

Figura 3. Gráfico de correlaciones de las variables y proyección de las variedades al plano de los dos primeros componentes. 


\section{Conclusiones}

- Los bastones de la variedad Frital INTA se caracterizan principalmente por los atributos que definen su textura tales como granulosidad, crocantez, firmeza y harinosidad. Por otro lado, Spunta está caracterizada con mayor longitud de bastón y por atributos como bastones húmedos, mayor aceitosidad residual y color más intenso. Kennebec tiene valores intermedios en humedad, granulosidad y aceitosidad residual. Shepody presenta una longitud de bastones similar a Spunta y con color externo más claro.

- La metodología utilizada permitió caracterizar sensorialmente las papas fritas de diferentes variedades, determinando diferencias significativas entre las mismas en descriptores sensoriales de textura y color.

- A efectos de determinar la influencia que tienen los atributos sensoriales de las distintas variedades en la aceptación por parte del consumidor, se deberán relacionar los resultados generados por los perfiles obtenidos por evaluadores entrenados con estudios de aceptabilidad en consumidores.

\section{Literatura Citada}

IRAM 20013. 1998. Análisis sensorial. Metodología. Perfil de textura.

IRAM 20019. 2004. Análisis sensorial. Metodología. Guía general para establecer un perfil sensorial. Esquema la (documento en estudio). $32 \mathrm{p}$.

Kabira, J.N. 2000. French fries and crisps processing characteristic of selected potatoes in Kenya. African Potato Association Conference Proceedings. African Potato Association 5: 507511

Kelly, A.R. y Scanlem, M.G. 2004. A fracture mechanics analysis of the texture of fried potato crust. Journal of Food Engineering 62: 417-423.
Kita, A. 2002. The influence of potato chemical composition on crips texture. Food chemistry 76 : 173-179.

Montauto-Graña, M.; Fernández-Fernández, E.; Vázquez-Odériz, M.L. y Ramera-Rodríguez, M.A. 2002. Development of a sensory profile for the specific denomination "Galicia potato". Food Quality and Preference 13: 99-106.

Quirien, E.A.; van Oschat, D. R. y Aked, J. 2003. Sensory characteristic of five sweet potato cultivars and their changes during storage under tropical conditions. Food Quality and Preference 14: 673-680.

Peña, D. 2002. Análisis de datos multivariantes. Mc Graw Hill, Madrid, 539 p.

R Development Core Team. 2004. R: A Language and environment for statistical computing. Foundation for Statistical Computing. Vienna, Austria.

Shomer, I. y Levy, D. 1988. Cell wall mediated bulkiness as related to the texture of potato (Solanum tuberosum L.) tuber tissue. Potato Research 31: 321-334.

Ross, K.A. y Scanlon, M.G. 2004. A fracture mechanics analysis of the texture of fried potato crust. Journal of Food Engineering 62: 417 423.

STATISTICA 5.1. 1997. Statsoft, Inc., Tulsa, USA.

Stone, H. y Sidel, J. 1993. Sensory Evaluation Practices. 2a edición. Academic Press, 338 p.

Trinchero, J.L.; Monti, M.C.; Cendoya, G. y Martínez, M.J. 2006. Perfiles sensoriales de variedades de papa. La Alimentación Latinoamericana 261: 60-65.

Van Oirschot, Q.E.A.; Rees, D. y Aked, J. 2003. Sensory characteristics of five sweet potato cultivars and their changes during storage under tropical conditions. Food Quality and Preference 14: 673-680. 